\title{
Manejo quirúrgico del divertículo de Zenker
}

\section{Surgical treatment of Zenker's diverticulum}

\author{
Enrique Rosales-Castañeda, Grecia C. García-Morán y José L. Martínez-Ordaz*
}

Servicio de Gastrocirugía, Hospital de Especialidades del Centro Médico Nacional Siglo XXI, Instituto Mexicano del Seguro Social, Ciudad de México, México

\section{Resumen}

Introducción: Los divertículos de Zenker son los divertículos más frecuentes del esófago. El tratamiento quirúrgico más utilizado es la diverticulectomía con miotomía del cricofaríngeo abierta. En años recientes se ha propuesto el tratamiento endoscópico. Objetivo: Presentar la experiencia en el manejo de esta patología en un hospital de referencia. Método: Fueron revisados los expedientes de los pacientes intervenidos quirúrgicamente por divertículo de Zenker en un periodo de 7 años. Los datos obtenidos incluyeron demográficos, cuadro clínico, abordaje diagnóstico, hallazgos transoperatorios y evolución posoperatoria. Resultados: Fueron intervenidos 10 pacientes durante este periodo, con una edad promedio de 64 años $( \pm$ 8); siete de ellos eran hombres. Los principales síntomas fueron regurgitación y disfagia, presentes en ocho y siete pacientes, respectivamente. Los estudios diagnósticos incluyeron endoscopia (nueve pacientes), esofagograma (seis pacientes) y manometría esofágica (tres pacientes). El tratamiento utilizado en todos los casos fue diverticulectomía con miotomía del cricofaríngeo. Las complicaciones incluyeron perforación esofágica transoperatoria (un paciente) y fístula esofágica (un paciente). Hubo resolución de los síntomas en nueve pacientes; el paciente restante ameritó nueva miotomía seis meses después, con una adecuada evolución. No hubo ninguna muerte. Conclusiones: La diverticulectomía con miotomía del cricofaríngeo es un tratamiento quirúrgico seguro para los pacientes con divertículo de Zenker.

PALABRAS CLAVE: Divertículo faringoesofágico. Divertículo de Zenker. Divertículo faringoesofágico por pulsión.

\section{Abstract}

Background: Zenker's diverticulum represents the most common diverticulum of the esophagus. The standard surgical treatment consists of open cricopharyngeal myotomy with diverticulectomy. In recent years endoscopic treatment has been proposed. Objective: We present the surgical experience of this disease in a referral hospital. Method: We reviewed the clinical records of all patients submitted to open surgical treatment for Zenker's diverticulum in a 7-year period. The retrieved information included demographic data, clinical manifestations, diagnostic approach, surgery findings and postoperative evolution. Results: During this period 10 patients were submitted to open surgical treatment; the mean age was 64 ( \pm 8) years and seven of them were male. The main complaint was regurgitation and dysphagia that was present in eight and seven patients respectively. Diagnostic studies included endoscopy (nine patients), esophagogram (six patients) and esophageal manometry (three patients). All underwent open cricopharyngeal myotomy with diverticulectomy. Complications included intraoperative esophageal perforation (one patient) and postoperative esophageal fistula (one patient). Resolution of the symptomatology occurred in 9 patients, the other patient required a new myotomy six months later with good results. There was no mortality. Conclusions: Cricopharyngeal myotomy with diverticulectomy is a safe option for patients with Zenker's diverticulum.

KEY WORDS: Zenker's diverticulum. Pharyngoesophageal diverticulum. Pharyngoesophageal pulsion diverticula.

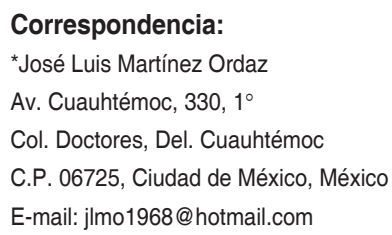

Fecha de recepción: 05-04-2018

Fecha de aceptación: 20-04-2018 DOI://dx.doi.org/10.24875/CIRU.M18000054
Cir Cir. 2018;86:355-358

Contents available at PubMed www.cirugiaycirujanos.com 


\section{Introducción}

Los divertículos esofágicos son poco comunes, con una prevalencia del 0.06 al $3.6 \%$ según series endoscópicas o radiológicas ${ }^{1-3}$.

Los divertículos que con mayor frecuencia se presentan son los divertículos de Zenker, descritos por Abraham Ludlow ${ }^{4}$ en 1769 y detallados de gran manera por Friedrich Albert von Zenker ${ }^{5}$ un siglo después. Tienen una frecuencia más elevada en las personas mayores de 50 años, y son dos a tres veces más frecuentes en el sexo masculino ${ }^{6,7}$. El cuadro clínico muestra una gran diversidad, desde escasos síntomas hasta pacientes con sintomatología sumamente incapacitante, que incluso puede llevar a un estado de desnutrición grave.

Las principales opciones terapéuticas son la miotomía del cricofaríngeo, la diverticulopexia con miotomía del cricofaríngeo y, la más utilizada, la diverticulectomía y miotomía del cricofaríngeo.

El objetivo de este estudio es describir la experiencia quirúrgica en un hospital de tercer nivel en el manejo del divertículo de Zenker durante un periodo de 7 años.

\section{Método}

Se realizó una revisión retrospectiva de todos los pacientes intervenidos quirúrgicamente en un hospital de tercer nivel por divertículo de Zenker en el periodo comprendido del 1 de enero de 2009 al 31 de diciembre de 2015. Una vez detectados, fueron revisados sus datos demográficos (edad, sexo y comorbilidad, entre otros), cuadro clínico de la enfermedad, estudios preoperatorios realizados relacionados con el padecimiento (esofagograma baritado, endoscopia, manometría esofágica), tratamiento quirúrgico realizado, complicaciones posoperatorias y seguimiento posoperatorio.

Debido al número de pacientes encontrado, se realizó como análisis estadístico, para las variables continuas, medidas de tendencia central y dispersión, y para las variables nominales se determinaron frecuencias, razones y proporciones (el porcentaje fue omitido, ya que son 10 pacientes).

\section{Resultados}

Durante el periodo de estudio se reportaron $10 \mathrm{pa-}$ cientes intervenidos quirúrgicamente por divertículo de Zenker. La edad media fue de $64( \pm 8)$ años. Siete eran varones y tres eran mujeres. La comorbilidad más frecuente fue hipertensión arterial en cuatro pacientes, seguida de diabetes mellitus en tres. Un paciente tenía antecedente de manejo endoscópico del divertículo con engrapadora transoral.

Los síntomas más frecuentes fueron regurgitación en ocho pacientes, disfagia en siete, halitosis en cuatro y sensación de cuerpo extraño en tres. Cuatro pacientes fueron catalogados previamente con sintomatología relacionada con reflujo gastroesofágico. El signo de Boyce (al desplazar la laringe en forma lateral se provocan ruidos hidroaéreos) y el signo de Quinn (regurgitación de comida hacia la boca por compresión externa de la bolsa) no fueron encontrados en nuestros pacientes ${ }^{9}$.

La endoscopia preoperatoria fue el estudio diagnóstico más utilizado, en nueve pacientes; se realizó esofagograma baritado en seis, tomografía computada en tres y manometría esofágica en tres.

En todos los pacientes se llevó a cabo diverticulectomía y miotomía del cricofaríngeo a través de una incisión cervical izquierda. La diverticulectomía fue realizada con engrapadora en todos los casos. El promedio de tiempo transoperatorio fue de $99( \pm 19)$ minutos, y el de la hemorragia transoperatoria fue de 41 ( \pm 34$) \mathrm{ml}$. El tamaño del divertículo fue, en promedio, de $3.1( \pm 1) \mathrm{cm}$. Durante el transoperatorio, la única complicación fue una perforación esofágica (manejada con cierre primario y gastrostomía) (Fig. 1).

Durante la evolución posoperatoria, un paciente desarrolló una fístula esofagocutánea de bajo gasto, la cual fue manejada de manera conservadora (nutrición parenteral y ayuno), y el paciente fue egresado sin complicaciones. En nueve pacientes hubo resolución de la sintomatología; el otro paciente presentó

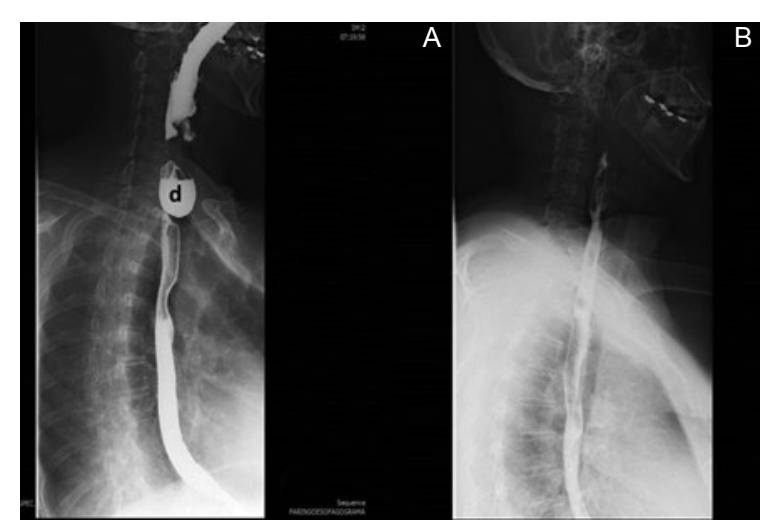

Figura 1. A: esofagograma baritado en el que se observa la imagen de un divertículo de Zenker (d). B: esofagograma baritado posoperatorio tras miotomía del cricofaríngeo y diverticulectomía. 
recurrencia de la sintomatología y se sospechó una miotomía del cricofaríngeo corta, por lo que requirió reintervención quirúrgica a los 6 meses, en la que se le realizó una nueva miotomía del cricofaríngeo y tuvo una adecuada evolución posoperatoria. No hubo ninguna muerte en este grupo de pacientes.

\section{Discusión}

La prevalencia de los divertículos esofágicos en la población general se considera que oscila entre el 0.01 y el $0.11 \%{ }^{10}$. Algunas publicaciones mencionan una distribución geográfica de esta afección, aunque sin especificar las causas, y destacan que es muy poco frecuente en Japón e Indonesia, en tanto que en el norte de Europa es más frecuente respecto del sur de este continente. En los EE.UU., Canadá y Australia es habitual y con una distribución más homogénea. Un estudio llevado a cabo en el Reino Unido estima una incidencia anual de 2 casos por cada 100,000 habitantes ${ }^{11}$. En México no se encuentra un reporte de incidencia concreto, y los pocos estudios realizados hacen alusión a una incidencia baja, como en el Hospital General de México, con una incidencia del 0.04\%, posterior a documentar tres casos en 5 años ${ }^{12}$.

La fisiopatología no se encuentra totalmente dilucida, pero dos teorías son las más aceptadas: la propuesta por Westrin, et al. ${ }^{13}$ refieren como causa la debilidad de la musculatura de la pared faríngea posterior adyacente al esfínter esofágico superior, y la postulada por Jackson y Shallow ${ }^{14}$ atribuye la formación del divertículo a la incoordinación del músculo cricofaríngeo en el momento de la deglución.

La dificultad deglutoria puede ser percibida por el paciente como un trastorno leve, inconstante y vinculado al tipo de ingesta durante el primer y el segundo tiempos de la deglución, denominados orofaríngeo y faríngeo, respectivamente, y a medida que aumenta el tamaño del divertículo se hace mayor y constante la dificultad deglutoria. En un estado avanzado, la imposibilidad para el paso del bolo tiene su origen en la compresión que ejerce la bolsa diverticular en la luz del esófago.

Se describen otros síntomas, como regurgitación, disnea por microaspiraciones o por efecto de la compresión de la vía aérea cuando el divertículo adquiere gran tamaño, halitosis y pérdida de peso. En este estudio, la regurgitación fue el principal síntoma referido por los pacientes, seguido de la disfagia y la halitosis.

Las complicaciones más frecuentes son cuadros de infecciones de vías respiratorias por aspiración del contenido diverticular, perforación que puede llegar a producirse por endoscopia o por la colocación de una sonda, y otras como fístula traqueoesofágica, hemorragia y parálisis de las cuerdas vocales.

La posible malignización del divertículo debe de ser sospechada en los pacientes con agravamiento de la disfagia, dolor intenso, episodios de hemoptisis e incremento de la intensidad de las regurgitaciones. El primer autor que la describió fue Schlesinger (citado por Halstead en 1903). Afecta a varones con una edad media mayor de 50 años. En 1969, Wychulis describió tres casos en una serie de 961 pacientes, con una incidencia del $0.3 \%$. Hoover encontró dos casos en una serie de 185 pacientes, con una incidencia del $1.1 \%$, 12 .

El estudio con ingestión de contraste permite apreciar la forma, el tamaño y la ubicación del divertículo. La endoscopia tiene un papel controversial, dado que algunos autores mencionan que es innecesaria e incluso riesgosa, mientras que otros opinan que es complementaria al permitir evaluar la posibilidad de cambios estructurales en la mucosa del divertículo. La manometría esofágica puede demostrar la asociación del divertículo con una respuesta anormal del músculo cricofaríngeo a la deglución o incluso con hipertonía de este. Sin embargo, debido a la anatomía (esfínter muy corto) y la fisiología (movimiento del esfínter durante la deglución), la evaluación es muy complicada ${ }^{15}$.

La miotomía del cricofaríngeo es el principal componente del tratamiento quirúrgico o endoscópico. Debe realizarse aun sin resultados de manometría esofági$\mathrm{ca}^{16}$. La recurrencia de la sintomatología o del divertículo, y las complicaciones posoperatorias (p. ej., fístula), están relacionadas muy frecuentemente con una miotomía incompleta. Este fue el tratamiento realizado en todos nuestros pacientes. La diverticulectomía resuelve mejor la sintomatología relacionada con la disfagia en comparación con la miotomía sola y con la diverticulopexia con miotomía ${ }^{16}$.

La diverticulotomía endoscópica es una técnica descrita por Mosher ${ }^{17}$ en 1917, abandonada por la mediastinitis que producía, y retomada en 1951 por Dohlman ${ }^{16}$ con buenos resultados. Consiste en dividir el septum entre el esófago cervical y la bolsa diverticular. Se puede realizar con electrocauterio, láser y, más recientemente, con engrapadora. Su principal ventaja es el tiempo quirúrgico corto y el rápido retorno a la vía oral. Actualmente no está muy difundida. A uno de nuestros pacientes se le había realizado, con una evolución no satisfactoria. 
La recurrencia en los pacientes con divertículo de Zenker manejados quirúrgicamente es del $16 \%{ }^{18}$. Como ya se comentó, completar la miotomía del cricofaríngeo suele ser suficiente para el tratamiento de la recurrencia del divertículo. La mortalidad actual es del $1-2 \%$.

\section{Conclusiones}

Los divertículos de Zenker son un diagnóstico poco frecuente. En México existe poca información sobre su prevalencia y sobre la experiencia de centros de atención de alta especialidad médica. Su sintomatología suele ser vaga. Los divertículos presentados en este estudio tienen una presentación semejante a los descritos en la literatura internacional en cuanto a características clínicas y estudios diagnósticos. El tratamiento utilizado en todos los casos fue diverticulectomía con miotomía del cricofaríngeo, que es el más recomendado actualmente.

\section{Responsabilidades éticas}

Protección de personas y animales. Los autores declaran que para esta investigación no se han realizado experimentos en seres humanos ni en animales.

Confidencialidad de los datos. Los autores declaran que han seguido los protocolos de su centro de trabajo sobre la publicación de datos de pacientes.

Derecho a la privacidad y consentimiento informado. Los autores declaran que en este artículo no aparecen datos de pacientes.

\section{Conflicto de intereses}

Los autores refieren no tener ningún conflicto de intereses con respecto a este trabajo.

\section{Bibliografía}

1. Hoghooghi D, Coakley F V, Breiman RS, Qayyum A, Yeh BM. Frequency and etiology of midesophageal diverticula at barium esophagography. Clin Imaging. 2006;30:245-7.

2. Watanabe S, Matsuda K, Arima K, Uchida Y, Nishioka M, Haruo T, et al. Detection of subclinical disorders of the hypopharynx and larynx by gastrointestinal endoscopy. Endoscopy. 1996;28:295-8.

3. Ekkberg O, Wahlgren L. Disfunction of pharyngeal swallowing. A cineradiographic investigation. Acta Radiol. 1985;26:389-95.

4. Ludlow A. A case of obstructed deglutition from a preternatural dilatation of, and bag formed in, the pharynx. Med Observ Enq. 1769;3:85-101.

5. Zenker FA, von Ziemssen H. Krankheiten des Oesophagus. En: von Ziemssen $\mathrm{H}$, editor. Handbuch der speciale Pathologie und Therapie. Leipzig, Germany: FC Vogel; 1877. p. 1-87.

6. Madick S. Perioperative care of the patient with Zenker's diverticulum. AORN J. 2001;73:904-13.

7. Onwugbufor MT, Obirieze AC, Ortega G, Allen D, Cornwell EE $3^{\text {rd }}$, Fullum TM. Surgical management of esophageal diverticulum: a review of the Nationwide Inpatient Sample database. J Surg Res. 2013;184:120-5.

8. Bonafede JP, Lavertu P, Wood BG, Eliacar I. Surgical outcome in 87 patients with Zenker's diverticulum. Laryngoscope. 1996:107:720-5.

9. Morales GD, Casanova RD, Garcia PM, Rodilla IG, Naranjo GA. Carcinoma epidermoide sobre un divertículo de Zenker. An Med Intern. 2002;19:27-30.

10. Watemberg S, Landau O, Avrahami R. Zenker's diverticulum: reappraisal. Am J Gastroenterol. 1996;91:1494-8.

11. Siddiq MA, Sood S, Strachan D. Pharyngeal pouch (Zenker's diverticulum). Postgrad Med J. 2001;77:506-11.

12. Pérez TE, Pérez PJ, Bernal SF, Abdo FJM, Murguía DD. Diagnóstico y tratamiento del divertículo de Zenker. Rev Med Hosp Gen Mex. 2005;68:155-9.

13. Westrin KM, Ergum S, Carsloo B. Zenker's diveticulum: a historical review and trends in therapy. Acta Otolaryngol (Stock). 1996;116:351-60.

14. Jackson C, Shallow TA. Diverticula of the esophagus: pulsion, traction, malignant and congenital. Ann Surg. 1926;83:1-19.

15. Sonbare DJ. Pulsion diverticulum of the oesophagus: more than just an out pouch. Indian J Surg. 2015;77:44-8.

16. Constantin A, Mates IN, Predescu D, Hoara P, Achim FI, Constantinoiu S. Principles of surgical treatment of Zenker diverticulum. J Med Life. 2012;5:92-7.

17. Mosher H. Webs and pouches of the oesophagus, their diagnosis and treatment. Surg Gynecol Obstet. 1917;25:175.

18. Skinner KA, Zuckerbraun L. Recurrent Zenker's diverticulum. Treatment with crycopharyngeal myotomy. Am Surg. 1998;64:192-4. 